\title{
Que política cultural é essa? Reflexões sobre a gestão pública da cultura nas universidades estaduais do Brasil ${ }^{1}$
}

\author{
¿Qué política cultural es esa? Reflexiones sobre la gestión pública de la \\ cultura en las universidades estaduales (subnacionales) de Brasil
What kind of cultural policy is that? Reflections on the public management of culture in state universities of Brazil

Juan Ignacio Brizuela ${ }^{2}$

Simonne Teixeira ${ }^{3}$

\begin{abstract}
Resumo
Para os estudiosos das políticas culturais é um grande desafio pensar em como se elabora a política cultural de uma universidade e, especialmente, como se materializa e se torna efetiva em seu território. Quem define as linhas estratégicas e as diretrizes de ação culturais em uma universidade? O dirigente municipal, estadual ou federal da pasta de educação e/ou cultura do governo ou o diretor do equipamento cultural universitário, geralmente associado à extensão? Poderia ser, ainda, o pró-reitor de extensão e, em algumas instituições de ensino superior, o pró-reitor de cultura que assume esse papel? Acaso não seria o próprio reitor o verdadeiro gestor cultural, com capital político e simbólico suficiente para orientar o desenvolvimento cultural da universidade? Nesse sentido, questionamos ainda: qual o papel dos centros, faculdades e laboratórios? Dos professores, estudantes, funcionários técnicos e trabalhadores comunitários? E em relação ao restante da comunidade e grupos artísticos, que influência exercem nas ações (e omissões) da universidade no campo cultural? Buscamos neste artigo esboçar algumas respostas a essas questões, não apenas considerando a noção de cultura acionada (seja sociológica ou antropológica), mas também discutindo a própria definição de política cultural e, consequentemente, as teorias e metodologias utilizadas para delimitar o escopo e abrangência do objeto de pesquisa. No caso das universidades estaduais, buscamos compreender as particularidades da gestão pública da cultura universitária e os processos de elaboração de políticas culturais nessas instituições de ensino superior do Brasil.
\end{abstract}

Palavras-Chave: gestão pública da cultura; política cultural universitária; território; universidades estaduais do Brasil.

\footnotetext{
${ }^{1}$ Artigo apresentado no Simpósio Temático 04 - Políticas públicas, diversidade cultural e descolonização, durante o II Seminário Latino-Americano de Estudos em Cultura - SEMLACult em Foz do Iguaçu/PR, Brasil, 2018.

${ }^{2}$ Doutor em Cultura e Sociedade (IHAC/UFBA); Bolsista recém doutor (FAPERJ) no Programa Interdisciplinar de Pós-graduação em Políticas Sociais; Casa de Cultura Villa Maria, Universidade Estadual do Norte Fluminense Darcy Ribeiro - UENF; Campos dos Goytacazes, Rio de Janeiro, Brasil; juanbrizuela.gpc@gmail.com.

${ }^{3}$ Doutora em Filosofía i Lletras (Historia) - Universitat Autònoma de Barcelona (Espanha); Diretora da Casa de Cultura Villa Maria - UENF; Professora e pesquisadora no Laboratório de Estudos do Espaço Antrópico, Centro de Ciências do Homem, da Universidade Estadual do Norte Fluminense Darcy Ribeiro, Brasil - UENF; Campos dos Goytacazes, Rio de Janeiro, Brasil; simonne@ @p.cnpq.br.
} 


\begin{abstract}
Resumen
Es un gran desafío para los estudiosos de las políticas culturales pensar en cómo se elabora la política cultural de una universidad y, especialmente, como se materializa y se torna efectiva en su territorio. ¿Quién define las líneas estratégicas y las directrices de acción culturales en una universidad? ¿El dirigente municipal, estadual (subnacional) o federal del área de educación y/o cultura del gobierno? ¿El director del equipamiento cultural universitario, generalmente asociado al área de extensión? ¿Podría ser el propio secretario académico de extensión y, en algunas instituciones de educación superior, el secretario académico de cultura de la universidad que asume ese papel? ¿Acaso no sería el propio rector el verdadero gestor cultural, con capital político y simbólico suficiente para orientar el desarrollo cultural de una universidad? En este sentido, cuestionamos: ¿cuál es el papel de los centros, facultades y laboratorios académicos? ¿De los profesores, estudiantes, funcionarios técnicos y trabajadores comunitarios? Y en relación al resto de la comunidad y grupos artísticos, ¿qué influencia ejercen tanto en las acciones como en las omisiones de la universidad en el campo cultural? Buscamos en este artículo esbozar algunas respuestas a esas cuestiones, considerando no solo la noción de cultura accionada (sea más sociológica o antropológica), sino también discutiendo la propia definición de política cultural y, consecuentemente, las teorías y metodologías utilizadas para delimitar el alcance y amplitud del objeto de investigación. En el caso de las universidades estaduales (subnacionales), buscamos comprender las particularidades de la gestión pública de la cultura y de los procesos de elaboración de políticas culturales en estas instituciones de educación superior de Brasil.
\end{abstract}

Palabras claves: gestión pública de la cultura; política cultural universitaria; territorio; universidades estaduales (subnacionales) de Brasil.

\begin{abstract}
For the scholars of cultural policies is a big challenge to think how a university's cultural policy is developed and, specially, how it is materialized, becoming effective in its territory. Who defines the strategies and guidelines of cultural actions to a university? Is the municipal, state (subnational) or federal leader of government's education office? Or the director of the university cultural unity, generally attached to university outreach? It could be yet the Provost for University Outreach, and in some higher education institutions, the Provost for Culture take over this role? By chance, would not the chancellor himself be the real cultural manager, with political and symbolic capital to lead the university's cultural development? This way, we still argue: what are the roles of the centers, faculties and laboratories? What are the roles of professors, students, technical workers and community workers? And relating to the rest of the community and artistic groups, how much influence they put into the university's actions (and neglects) within the cultural field? We are looking in this paper to draw some answers to these questions, not only considering the notion of culture that is chosen (whether the sociological or the anthropological approach), but also discussing the definition of cultural policy. Consequently, theories and methodologies are used to define the focus and the range on the object of research too. In the case of state universities, we aim to comprehend the particularities of cultural public management in universities and the processes of elaboration of cultural policies inside the brazilian higher education institutions.
\end{abstract}

Keywords: cultural public management; university cultural policy; territory; brazilian state universities

\title{
1. Introdução
}

Embora existam antecedentes de programas, experiências e pesquisas no campo das políticas culturais universitárias, não é tão fácil visualizar e muito menos definir os parâmetros que caracterizam a política cultural de uma instituição de ensino superior. Neste caso, nossa reflexão se inicia pela ausência (aparente) de um conjunto de intervenções em arte e cultura, de forma sistemática e continua, na Universidade Estadual do Norte Fluminense Darcy Ribeiro - UENF. O questionamento mais recorrente, conforme ainda podemos escutar em diferentes âmbitos, é de que política cultural vamos falar, se na universidade isso não existe. 
Diante deste cenário, nos propusemos mapear algumas iniciativas de universidades estaduais do Brasil, localizadas preferencialmente fora das capitais, que se fossem aplicadas todas juntas na mesma instituição de ensino superior não estaríamos longe de caracterizar como uma efetiva política cultural universitária. Esses elementos são: a) equipamento cultural específico; b) formação em arte e cultura na graduação; c) cursos de especialização ou pósgraduação na área de organização da cultura e; d) institucionalização de um plano de cultura universitário.

Registramos, assim, iniciativas e projetos em andamento da Universidade Estadual de Londrina (PR), da Universidade Estadual de Montes Claros (MG), da Universidade Estadual de Santa Cruz (BA) e da Universidade Estadual de Maringá (PR), entre outras, no sentido de ir preenchendo várias das lacunas que a UENF e outras instituições de ensino superior poderiam ter na elaboração de execução de uma política cultural universitária a nível estadual no Brasil.

\section{Dimensões da política cultural universitária e o planejamento estratégico}

As universidades estatais - sejam federais, estaduais ou municipais - são agentes públicos com características híbridas entre Estado, sociedade civil e comunidade, de forma semelhante ao que acontece com os conselhos participativos de políticas públicas (RUBIM; BRIZUELA e LEAHY, 2010). Assim sendo, a depender da noção de cultura acionada - seja uma visão sociológica mais restrita ou aquela vinculada a uma perspectiva antropológica mais ampla - os atores, funções e expectativas vão mudar.

Para Isaura Botelho (2001), do ponto de vista da política pública, é diferente trabalhar com cultura no seu sentido mais amplo da sociedade, do que com o circuito organizado das artes e da produção cultural mais específica. A partir das categorias analíticas utilizadas pelo sociólogo chileno José Joaquín Brunner, a pesquisadora propõe trabalhar com duas dimensões da cultura na gestão pública, a antropológica e a sociológica:

Na dimensão antropológica, a cultura se produz através da interação social dos indivíduos, que elaboram seus modos de pensar e sentir, constroem seus valores, manejam suas identidades e diferenças e estabelecem suas rotinas. Desta forma, cada indivíduo ergue à sua volta, e em função de determinações de tipo diverso, pequenos mundos de sentido que lhe permitem uma relativa estabilidade (p. 74, grifo nosso).

Nesta perspectiva, se incluem significados e práticas vinculadas a origens regionais, interesses profissionais ou econômicos, esportivos e sociais, de sexo, origem étnica, geração etc. A forma em que a gestão das políticas culturais poderia intervir concretamente nesta 
dimensão antropológica é a partir de uma reorganização das estruturas sociais e de uma profunda distribuição dos recursos econômicos. Para Botelho, é preciso uma mudança radical de médio e longo prazo que possa interferir em hábitos e costumes arraigados, nas formas de trabalho, no uso do tempo livre, enfim, nas relações de vizinhança e sociabilidade em sentido amplo.

Por outro lado, a dimensão sociológica das políticas públicas de cultura compreende um âmbito especializado de atuação com uma intencionalidade explícita, meios de produção, públicos e canais determinados:

[...] a dimensão sociológica da cultura refere-se a um conjunto diversificado de demandas profissionais, institucionais, políticas e econômicas, tendo, portanto, visibilidade em si própria. Ela compõe um universo que gere (ou interfere em) um circuito organizacional, cuja complexidade faz dela, geralmente, o foco de atenção das políticas culturais, deixando o plano antropológico relegado simplesmente ao discurso (BOTELHO, 2001, p. 74, grifo nosso).

Trata-se de um sistema socialmente organizado muito mais visível, que contém uma formação e aperfeiçoamento específico, programas e agencias de financiamento com critérios e escopos determinados. Enfim, se materializa em um circuito organizado que fomenta a produção, circulação e consumo de bens simbólicos, ou seja, aquilo que o senso comum entende por cultura, incluindo as expressões artísticas em sentido estrito. Por estas características, a investigadora afirma que esta dimensão termina sendo privilegiada pelas ações de governo, deixando o plano antropológico para discursos sem efetividade prática. Para atingir essa outra dimensão mais ampla seria necessário um esforço coordenado e articulado do governo, especialmente a nível municipal, local apontado pela autora como plano privilegiado de atuação e de produção dos sentidos cotidianos.

A maior parte das ações e práticas universitárias que veremos neste artigo estão dentro desta dimensão sociológica das políticas públicas de cultura, mas acreditamos que é importante pelo menos registrar essa dimensão mais ampla e tentar refletir sobre como se territorializa uma política cultural universitária na perspectiva antropológica. Enquanto forma de distribuição dos recursos sociais e econômicos, as políticas de cotas poderiam ser um exemplo desta política pública universitária em sentido mais amplo. Aliás, experiências de vestibular indígena como acontecem na Universidade Estadual de Campinas (UNICAMP, 2018) podem ser considerados políticas culturais na sua dimensão antropológica. 
Uma primeira aproximação à política cultural universitária no Brasil parece indicar uma visão mais patrimonial nas ações e programas culturais das instituições de ensino superior, limitada às linguagens artísticas tradicionais. Contudo, analisando de forma histórica e mais ampla, vemos que existem diversas experiências que abrangem o Centro Popular de Cultura - CPC instituído em 1962 em Rio de Janeiro e associado à União Nacional de Estudantes - UNE até a mais recente criação de pró-reitoras específicas para o campo da cultura, como ocorre na Universidade Federal do Cariri - UFCA, passando pela convocatória "Mais cultura nas Universidades" de 2014 do Ministério de Cultura - MinC junto al Ministério de Educação - MEC, onde se estimula a formulação de planos de cultura nas instituições federais. Ainda mais, já existem iniciativas em universidades estaduais nesta linha como os Planos Institucionais de Cultura da Universidade Estadual de Campinas (UNICAMP, 2015), na região metropolitana de São Paulo, da Universidade Estadual de Maringá (UEM, 2017) no estado de Paraná e da Universidade do Estado do Rio Grande do Norte (UERN, 2018) no Nordeste.

Sendo assim, são diversas as experiências de planejamento estratégico nas instituições de ensino superior, mas não tão abundantes aquelas no campo da cultura e das artes, em especial nas universidades estaduais do Brasil. Em geral, as ações universitárias em arte e cultura não estão institucionalizadas, sistematizadas nem monitoradas. Neste sentido, o plano de cultura busca organizar e dar um marco de previsibilidade aos programas e ações culturais realizados pela universidade ao longo dos anos. O planejamento estratégico pretende facilitar a participação e transparência na construção de metas e indicadores culturais a partir das necessidades concretas da comunidade acadêmica e não acadêmica.

\section{Panorama das universidades estaduais do Brasil}

De acordo com o documento de trabalho do Núcleo de Pesquisas sobre Ensino Superior da Universidade de São Paulo (1998), até 1981 existiam apenas 9 universidades estaduais reconhecidas em todo o território brasileiro: quatro no Sudeste, a Universidade de São Paulo (USP, 1934), a Universidade do Estado do Rio de Janeiro (UERJ, 1953), a Universidade Estadual Paulista (UNESP, 1976) e a Universidade Estadual de Campinas (UNICAMP, 1976); três no Sul, a Universidade Estadual de Londrina (UEL, 1971), a Universidade Estadual de Ponta Grossa (UEPG, 1973) e a Universidade Estadual de Maringá (UEM, 1976); e dois no Nordeste, a Universidade Estadual do Ceará (UECE, 1977) e a 
Universidade Estadual do Maranhão (UEMA, 1981). Em 1996, esse número aumenta consideravelmente chegando a 31 instituições de ensino superior estaduais. O Censo de Ensino Superior do Brasil de 2016 registra 40 universidades mantidas pelos estados do país, uma delas sendo a Fundação Centro Universitário Estadual da Zona Oeste - UEZO no Rio de Janeiro. Poderíamos somar também a Fundação Universidade Aberta do Distrito Federal FUNAB com sede em Brasília, e a mais recentemente Universidade Estadual da Região Tocantina do Maranhão - UEMASUL, com sedes nas de Imperatriz e Açailândia do Estado de Maranhão. Contudo, vale registrar que estas duas últimas não aparecem formalmente nos dados do MEC disponibilizados pelo INEP-Data.

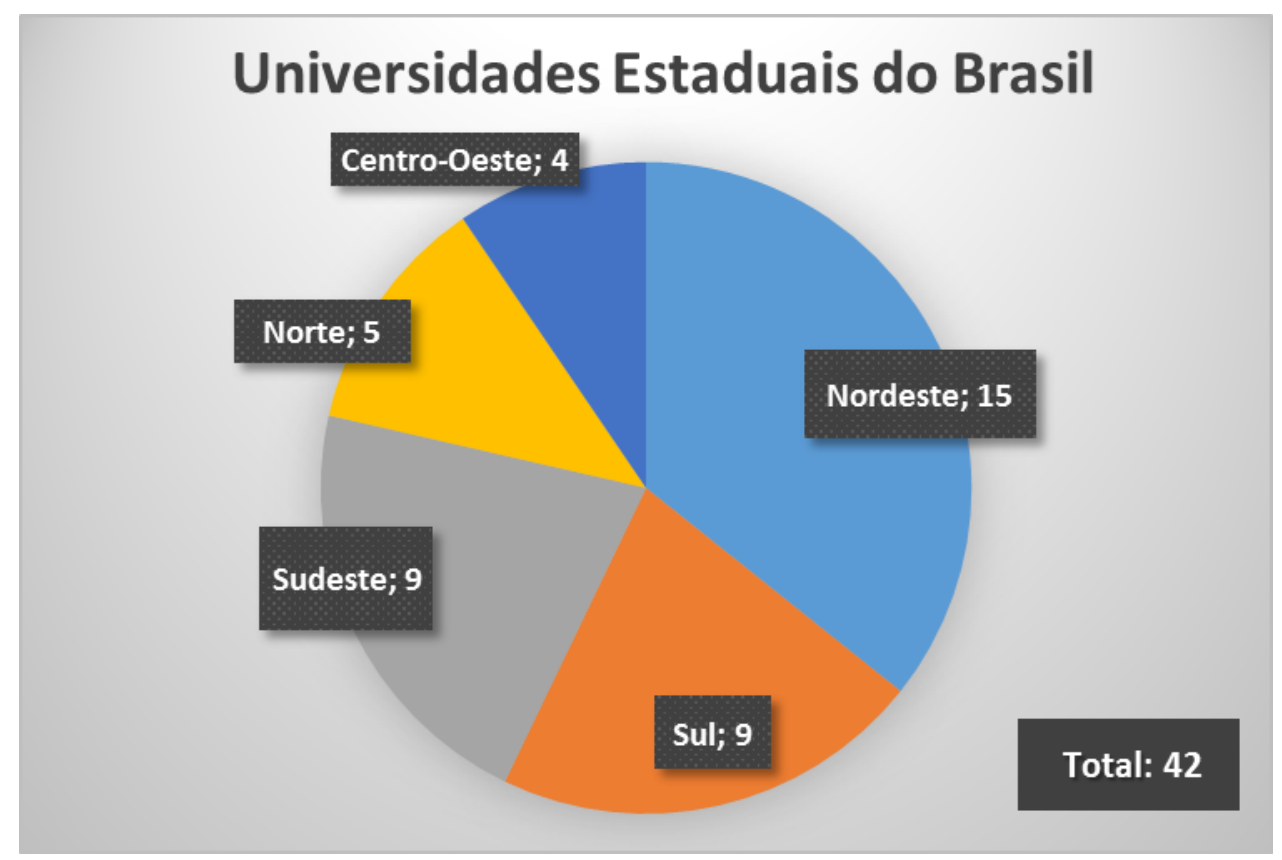

Figura 1 - Distribuição das universidades estaduais por regiões Fonte: Elaboração própria com dados do INEP-Data (2018).

Em 2016 tinha mais de 8 milhões de alunos matriculados em todas as instituições de ensino superior do Brasil. Um $75 \%$ estudo no âmbito privado, razão pela qual aproximadamente 2 milhões de matrículas são da rede pública. As universidades estaduais assumem parte importante das matrículas das instituições públicas, com mais de 600 mil alunos vinculados. Desse total, mais de 180 mil pessoas estudam nas universidades paulistas USP, UNESP e UNICAMP. Além disso, das 42 instituições estaduais que registramos em 2018, 22 têm sua sede principal no interior do país, conforme pode ser visualizado na tabela 1. 
RELACult - Revista Latino-Americana de Estudos em Cultura e Sociedade

Revista Latinoamericana de Estudios en Cultura y Sociedad | Latin American Journal of Studies in Culture and Society V. 05, ed. especial, mai., 2019, artigo no 1543 | claec.org/relacult | e-ISSN: 2525-7870

Tabela 1 - Universidades estaduais no interior/capital por região

\begin{tabular}{|c|c|c|c|c|c|c|c|}
\hline Região/IES & Interior & Capital & Total & Região/IES & Interior & Capital & Total \\
\hline Centro-Oeste & $\underline{\mathbf{3}}$ & 1 & 4 & Norte & $\underline{\mathbf{0}}$ & 5 & 5 \\
\hline FUNAB & & $\mathrm{C}$ & & UEA & & $\mathrm{C}$ & \\
\hline$\underline{\mathrm{UEG}}$ & I & & & UEAP & & $\mathrm{C}$ & \\
\hline$\underline{\text { UEMS }}$ & I & & & & & & \\
\hline UNEMAT & I & & & UEP A & & $\mathrm{C}$ & \\
\hline Nordeste & $\underline{9}$ & 6 & 15 & OL1 & & C & \\
\hline UECE & & $\mathrm{C}$ & & UNITINS & & $\mathrm{C}$ & \\
\hline$\underline{\text { UEFS }}$ & I & & & Sudeste & $\underline{3}$ & 6 & 9 \\
\hline & & $\mathrm{C}$ & & UEMG & & $\mathrm{C}$ & \\
\hline UEMASUL & I & & & $\underline{\mathrm{UENF}}$ & I & & \\
\hline$\underline{\mathrm{UEPB}}$ & I & & & UERJ & & $\mathrm{C}$ & \\
\hline UERN & I & & & UEZO & & $\mathrm{C}$ & \\
\hline$\underline{\text { UESB }}$ & I & & & UNESP & & $\mathrm{C}$ & \\
\hline$\underline{\text { UESC }}$ & I & & & UNICAMP & I & & \\
\hline UESPI & & $\mathrm{C}$ & & UNIMONTES & I & & \\
\hline UNCISAL & & $\mathrm{C}$ & & UNIVESP & & $\mathrm{C}$ & \\
\hline UNEAL & I & & & USP & & $\mathrm{C}$ & \\
\hline UNEB & & $c$ & & Sul & $\underline{7}$ & 2 & 9 \\
\hline $\begin{array}{l}\text { UPE } \\
\text { URCA }\end{array}$ & I & $c$ & & UDESC & & $\mathrm{C}$ & \\
\hline$\overline{\mathrm{UVA}}$ & I & & & $\underline{\text { UEL }}$ & I & & \\
\hline & & & & $\underline{\text { UEM }}$ & I & & \\
\hline & & & & $\underline{\text { UENP }}$ & I & & \\
\hline & & & & $\underline{\mathrm{UEPG}}$ & I & & \\
\hline & & & & UERGS & & $\mathrm{C}$ & \\
\hline & & & & UNESPAR & I & & \\
\hline & & & & $\underline{\text { UNICENTRO }}$ & I & & \\
\hline & & & & UNIOESTE & I & & \\
\hline & & & & Total Geral & $\underline{22}$ & 20 & 42 \\
\hline
\end{tabular}

Fonte: Elaboração própria com dados do INEP-Data (2018). 


\section{Ações em arte e cultura das universidades estaduais - elementos de uma política cultural?}

Conforme registramos, é difícil encontrar uma política cultural universitária já sistematizada e em execução nas universidades estaduais do Brasil, muito mais nos interiores e fora das capitais. Contudo, mesmo nas situações mais críticas existem ações e iniciativas em arte e cultura em todas as universidades estaduais do país, algumas mais modestas e outras mais complexas. E se pensamos que o que define uma política cultural é precisamente esse conjunto de intervenções, seja por ação ou por omissão, resulta que podemos arriscar alguns elementos mínimos desta política cultural universitária a partir de experiências práticas que destacamos a continuação.

\subsection{Casas de Cultura Universitárias: Villa Maria da UENF (RJ) e a UEL (PR)}

A Universidade Estadual do Norte Fluminense Darcy Ribeiro - UENF existe desde 1993 e está fazendo 25 anos. Como unidade autárquica e autônoma a sua constituição é mais recente, apenas de 2002. São aproximadamente 5 mil alunos de graduação presencial, pósgraduação e de ensino à distância (EAD), distribuídos da seguinte forma: 1800 na graduação, 1200 na pós-graduação e quase 2000 no EAD.

A Casa de Cultura Villa Maria/CCVM da UENF situa-se em local nobre da cidade de Campos dos Goytacazes e compõe, junto ao Liceu de Humanidades, ao Palácio Nilo Peçanha (Câmara de Vereadores) e à praça do Jardim do Liceu, uma área de preservação cultural de grande beleza, conhecida como quadrilátero histórico. 


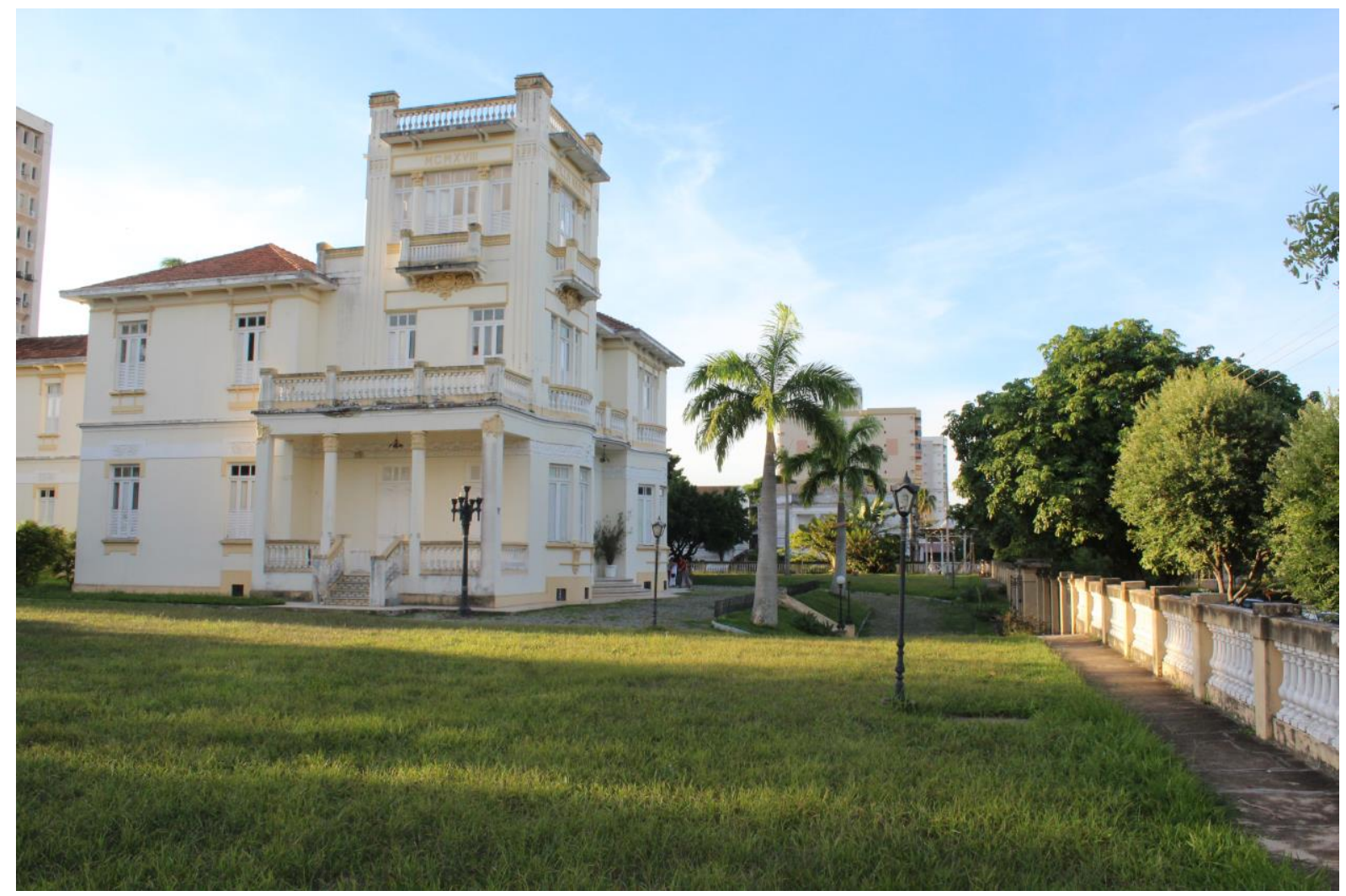

Fotografia 1 - Casa de Cultura Villa Maria da UENF Fonte: http://villamaria.uenf.br/sobre-a-villa/\#galeria

As práticas culturais, o ensino das artes e o incentivo à inventividade devem ser permanentemente estimulados no ambiente universitário, na mesma medida em que se estimula o conhecimento científico. Uma casa de cultura vinculada à instituição universitária deve desenvolver plenamente suas atribuições de ensino, pesquisa e extensão, tomando para si estas competências de modo indissociável. Assim, deve incorporar as mais diferentes manifestações da criatividade humana, evitando a mera reprodução das práticas culturais hegemônicas e de massas, diferenciando-se pela qualidade crítica e investigativa.

Estas ideias estão presentes na proposta elaborada pelo filósofo José Américo Pessanha, no início dos anos 1990, a pedido de Darcy Ribeiro para a Casa de Cultura Villa Maria. Em seu projeto, Pessanha assinala que a Villa Maria, "está projetada para a prestação de serviços à comunidade e ao mesmo tempo ser cartão de visitas da Universidade Estadual do Norte Fluminense" (1993, p. 179). Inspirados nesta proposição, foram criados os setores ${ }^{4}$

\footnotetext{
${ }^{4} \mathrm{O}$ espaço da Casa de Cultura abrigou, no momento de sua criação, os seguintes setores: Fonoteca, Videoteca, Hemeroteca e Sala de Leitura (que neste momento passam por uma reestruturação, buscando adequar as atividades desenvolvidas na Casa de Cultura às novas circunstâncias da universidade).
} 
que deram vida à Casa e que tinham por objetivo a promoção e a difusão da cultura para a comunidade local.

Neste sentido, se entende que a Casa de Cultura Villa Maria deve incentivar e fomentar atividades de pesquisa e produção cultural que devem configurar-se, como caminhos de aproximação entre a Universidade, a cidade e a região. No lugar de simplesmente abrigar exposições eventuais e seminários, ela mesma deve ser capaz de propor temas e análises sobre questões culturais convocando a comunidade universitária e regional ao mais amplo diálogo. A exemplo da proposta original da UENF, que direciona à experimentação - daí os laboratórios no lugar dos departamentos -, a Villa Maria deve situar-se como um espaço de experimentação, sobretudo no campo da cultura, que constitui sua natureza. Neste sentido, deve também constituir-se como locus privilegiado de pesquisa.

Desde sua criação a Casa de Cultura Villa Maria esteve ligada à difusão da música, do cinema e do incentivo à leitura, desenvolvidas nos setores então existentes - a Fonoteca, na Videoteca e na Sala de Leitura - que atendia gratuitamente o público em geral, acolhendo-o no que havia de expressivo nestes campos da arte/cultura, nacional e internacional, sem perder o foco para a produção local. A CCVM acolheu também o primeiro ponto de internet gratuita na cidade de Campos dos Goytacazes.

Enquanto equipamento cultural em sentido estrito, encontramos outra casa de cultura universitária em uma instituição estadual de ensino superior do interior de Paraná, com uma configuração diferente da CCVM da UENF.

A Universidade Estadual de Londrina - UEL foi criada em 1970 com a junção de cinco Faculdades e transformada em autarquia em 1991. Nesta IES estudam 16 mil alunos de graduação e pós-graduação. Apenas um ano depois a sua fundação, em 1971, surge a Casa de Cultura UEL que destacamos neste panorama.

Segundo informações do site oficial desta instituição (UEL, s.d.), a casa de cultura é responsável pela realização de importantes eventos culturais nas áreas de artes visuais, artes cênicas, música, cinema e vídeo com abrangência na cidade de Londrina e região. O seu principal objetivo é estimular a formação, produção e circulação artística e cultural, além de apoiar a produção regional e também nacional e internacional.

Chama a atenção a configuração institucional e territorial deste equipamento cultural universitário, instalado atualmente em seis espaços distintos: I. o Cine Teatro Universitário Ouro Verde UEL; II. a Casa Branca (Artes Plásticas); III. a Casa Tupi (Sede Administrativa, 
Centro de Documentação, Música); IV. o Barracão Vermelho (Artes Cênicas); V. a Sala Rômulo Veronesi (Edifício Julio Fuganti) e; VI. o Cine Com-Tour UEL.

Entra as várias ações e parcerias desta casa, se destacam o Festival Internacional de Londrina - FILO e o Festival de Música de Londrina - FML, a Orquestra Sinfônica da Universidade Estadual de Londrina - OSUEL e as orquestras Prelúdio e Jovem Sinfônica UEL, os projetos Vitrine das Artes, Foto Link, Cine Clube UEL e Rádio Fórum. Além disso, também disponibiliza ao público seu acervo de documentação sonora, impressa e audiovisual, possibilitando consultas e ações artísticas e formativas na Audioteca, Biblioteca, Videoteca e Cinemateca.

\subsection{Formação em arte e cultura na UNIMONTES (MG)}

Fundada em 1962 e com aproximadamente dez mil alunos de graduação presencial, pós-graduação e à distância (EAD), a Universidade Estadual de Montes Claros UNIMONTES (MG) se destaca pela formação em artes e cultura que oferece, tanto a nível de bacharelado quanto de licenciatura. Os cursos de graduação em Artes - Habilitação em Música, Artes - Habilitação em Teatro e Artes Visuais são importantes na construção de uma política cultural consistente na universidade e no desenvolvimento da região. Estes cursos, vinculados ao Centro de Ciências Humanas - $\mathrm{CCH}$, são um desmembramento de uma licenciatura antiga sobre Educação Artística e oferecem quase 100 vagas anualmente para a formação de professores nesta área (UNIMONTES, s.d.).

Nesta instituição existe uma coordenadoria em cultura, vinculada à pró-reitoria de extensão, que disponibiliza suporte às ações e projetos oriundos dos departamentos da universidade, que visem implementação de projetos nas áreas culturais; trabalha como interlocutora entre a comunidade e os departamentos para favorecer o fomento da área supracitada e; estuda estratégias e formas de recursos para viabilizar a implementação de projetos de arte e cultura, em consonância com os interesses da universidade.

\subsection{Especialização em gestão cultural na UESC (BA)}

Com faculdades em funcionamento desde os anos 1970, a Universidade Estadual de Santa Cruz - UESC (BA) foi oficializada em 1995 como autarquia e é a mais nova das 4 IES mantidas pelo Estado da Bahia, junto com a UNEB, a UFES e a UESB. São mais de 7 mil alunos de graduação presencial, pós-graduação e EAD. Quase 20 anos depois dessa formalização, a UESC concretiza uma iniciativa de especialização em gestão cultual que, pela sua especificidade, merece destaque neste percurso pelas universidades estaduais do Brasil. 
Seguindo as informações do site oficial desta instituição (UESC, s.d.), a Especialização em Gestão Cultural, lato sensu, foi autorizada em abril de 2015. Este curso resulta de ações de extensão em cultura, iniciadas em 2008, implementadas pela PROEX em parceria com distintas instancias da própria universidade - como o Departamento de Letras e Artes (DLA), o Núcleo de Artes da UESC (NAU) e o Ponto de Cultura Arte e Educação Musicalização e Canto Coral - como também parcerias da Secretaria de Cultura do Estado da Bahia, Fundação Cultural de Ilhéus, Associação dos Municípios da Região Cacaueira da Bahia (AMURC), prefeituras municipais do Território Litoral Sul da Bahia e Fórum de Agentes, Empreendedores e Gestores Culturais do Sul da Bahia (FAEG-SUL). Este último, inclusive, formalmente demandou à UESC um curso de especialização na área cultural.

O objetivo deste curso é a formação, em nível de Pós-Graduação, lato sensu, de profissionais atuantes no campo da cultura, em especial nas seguintes áreas: políticas culturais, marketing cultural, economia da cultura, legislação cultural brasileira, elaboração de projetos culturais, captação de recursos para a cultura, gestão cultural na esfera pública e privada, sistemas de cultura e redes colaborativas e, produção, distribuição e consumo de bens culturais.

\subsection{A construção do plano de cultura universitário na UEM (PR)}

A Fundação Universidade Estadual de Maringá - UEM (PR) também existe desde 1970, tornando-se uma autarquia em 1991. A UEM atende atualmente mais de 20 mil alunos de graduação presencial, pós-graduação e EAD. Das cinco universidades estaduais registradas neste trabalho, apenas a UEM conta com uma Pró-reitoria de Extensão e Cultura (PEC) e nos últimos três anos tem realizado um esforço explícito na construção de um Plano de Cultura universitário que seria quase que inédito para uma universidade estadual (UEM, 2017), especialmente de uma região não central.

Conforme registrados anteriormente, existem iniciativas similares na UNICAMP e na UERN. Contudo, nenhuma das três universidades estaduais disponibiliza de forma aberto e acessível estes planos aprovados e teoricamente em funcionamento. Isto dificulta enormemente o acompanhamento das metas, diretrizes e estratégias que possam estar registradas neste documento e, em especial, qual foi o diagnóstico cultural que levou a cada um dos objetivos registrados. 


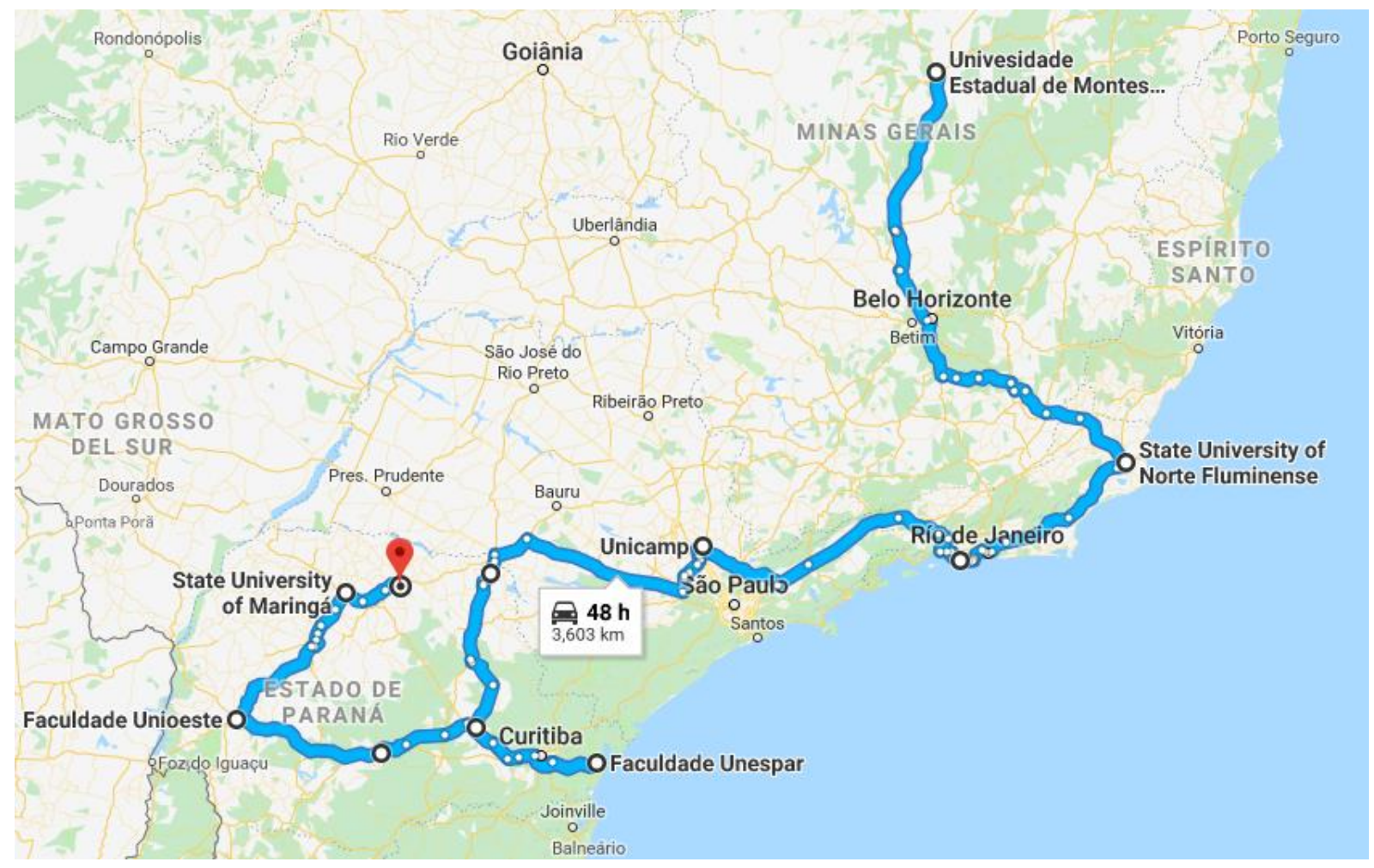

Cartografia 1 - Universidades estaduais do interior, regiões Sul e Sudeste Fonte: Google Maps, 2018.

\section{A final, o que define uma política cultural universitária a nível estadual?}

Esta pesquisa exploratória não pretende definir de forma rigorosa o que seria uma política cultural a nível estadual aplicável às dezenas de universidades estaduais que existem no país. Contudo, se consideramos e conectamos os elementos registrados anteriormente pelo menos um equipamento cultural específico, preferencialmente com ações desconcentradas e territorializadas; formação em arte e cultura na graduação e na pósgraduação; institucionalização cultural com hierarquia (ex. pró-reitoria) e em um plano de cultura universitário - já teremos andado um bom caminho que aproveita o melhor das práticas e experiências já existentes no Brasil.

Finalmente, a construção de circuitos culturais interuniversitários, pensando na dimensão sociológica das políticas públicas de cultura que discutimos no início do texto, se apresenta como um dos desafios mais urgentes nestes processos de fortalecimento da dimensão cultural nas universidades. O projeto Corredor Cultural Forproex Sudeste, os Fóruns de Gestão Cultural nas Universidades e a mais recente Teia Interuniversitária das Artes - TEIA são iniciativas positivas que precisam ser consolidadas também internamente 
em cada uma das instituições de ensino superior para possibilitar essa retroalimentação sinérgica das políticas culturais universitárias.

\section{Bibliografia}

BOTELHO, Isaura. Dimensões da cultura e políticas públicas. Revista São Paulo em perspectiva, São Paulo: Abr-Jun, Vol. 15, Nº 2, 2001, p. 73-83.

PESSANHA, José Américo. Casa da Cultura Villa Maria. In: Faculdade de Educação e Comunicação - Universidade do Terceiro Milênio, vol. 3. Campos dos Goytacazes: UENF, 1993. p. 179-190. Disponível em: http://www.uenf.br/portal/index.php/br/baixe-o-planoorientador-da-uenf.html Acesso em: 01 dez. 2018.

RUBIM, Iuri; BRIZUELA, Juan Ignacio; LEAHY, Renata. Políticas Culturais, Democracia e Conselhos de Cultura. In: RUBIM, Albino; FERNANDES, Taiane; RUBIM, Iuri. (Eds.). Políticas Culturais, Democracia e Conselhos de Cultura. Salvador: EDUFBA, 2010. p. 111144.

SAMPAIO, Helena; BALBACHESKY, Elisabeth; PEÑALOZA, Verónica. Universidades Estaduais no Brasil - Características Institucionais. Documento de trabalho 04/98. Núcleo de Pesquisas sobre Ensino Superior da Universidade de São Paulo. São Paulo: USP, 1998. Disponível em: http://nupps.usp.br/downloads/docs/dt9804.pdf Acesso em: 01 dez. 2018.

UEL. Casa de Cultura. [s.d.] Disponível em: http://www.uel.br/cc/ Acesso em: 01 dez. 2018.

UEM. Plano que vai definir a política cultural da UEM é entregue para a Reitoria. Notícias, 22 nov. 2017. Disponível em: goo.gl/SCZqAY Acesso em: 01 dez. 2018.

UERN. CONSEPE aprova o Plano Institucional de Cultura da UERN. 28 mar. 2018. Disponível em: http://portal.uern.br/blog/consepe-aprova-o-plano-institucional-de-cultura-dauern/ Acesso em: 01 dez. 2018.

UESC. Especialização em Gestão Cultural. [s.d.] Disponível em: http://www.uesc.br/cursos/pos_graduacao/especializacao/gestao_cultural/ Acesso em: $01 \mathrm{dez}$. 2018.

UNICAMP. Discutindo a política cultural da Unicamp com convidados internacionais e do MinC. 10 set. 2015. Disponível em:

http://www.unicamp.br/unicamp/noticias/2015/09/10/discutindo-politica-cultural-daunicamp-com-convidados-internacionais-e-do-minc Acesso em: 01 dez. 2018.

. Unicamp aplica seu primeiro Vestibular Indígena e faz história. 02 dez. 2018. Disponível em: https://www.unicamp.br/unicamp/noticias/2018/12/02/unicamp-aplica-seuprimeiro-vestibular-indigena-e-faz-historia Acesso em: $01 \mathrm{dez} .2018$.

UNIMONTES. Curso de Licenciatura em Artes Visuais. [s.d.] Disponível em: http://www.unimontes.br/index.php/centro-de-ciencias-humanas-cch/4760-artes-visuaismontes-claros Acesso em: 01 dez. 2018. 